\title{
Experiência preliminar com novo filtro de veia cava: resultados de 15 implantes
}

\author{
Preliminary experience with a new vena cava filter: \\ results of 15 implantations
}

\author{
Winston B. Yoshida ${ }^{1}$, Hamilton A. Rollo², Mariângela Giannini² ${ }^{2}$ Marcone L. Sobreira ${ }^{2}$, Regina Moura ${ }^{2}$

\section{Resumo} \\ Abstract
}

Este estudo apresenta resultados preliminares obtidos com um novo filtro permanente de veia cava, baseado no desenho de Greenfield, com três hastes prolongadas de um total de seis, para dar estabilidade central ao filtro na luz da veia cava. Neste artigo, relatamos sua avaliação clínica preliminar quanto à aplicabilidade, eficácia e segurança. De agosto de 2004 a dezembro de 2006, 15 filtros foram implantados em nove homens e seis mulheres, com idades variando de 38 a 79 anos (média de 57,8 anos). O acesso foi feito sempre por via transjugular. As indicações foram: trombose venosa proximal, com contra-indicação de anticoagulação em 12 pacientes; complicações hemorrágicas com anticoagulação em dois pacientes; e embolia pulmonar, apesar de anticoagulação adequada, em um paciente. Os filtros foram avaliados quanto à liberação, inclinação, mau posicionamento e perfuração de cava. No seguimento, avaliou-se trombose no local de acesso, tromboembolismo venoso recorrente, migração do filtro e trombose de cava pelo ultra-som. Nenhum paciente recebeu anticoagulantes no seguimento. $\mathrm{O}$ filtro foi liberado com sucesso em todos os casos sem mau posicionamento, inclinação, perfuração ou trombose de acesso. Os pacientes foram seguidos entre 3 e 23 meses (média de 11 meses). Nenhum paciente teve recorrência de tromboembolismo venoso. Não houve casos de trombose de veia cava ou migração do filtro. Óbito ocorreu em sete casos, todos relacionados com a moléstia de base. Os resultados preliminares indicam potencial eficácia e segurança do uso do novo filtro no período estudado.

Palavras-chave: Filtro de veia cava, desenho de próteses, embolia pulmonar/prevenção, controle, trombose venosa.

\section{Introdução}

Dentre os filtros permanentes de veia cava disponíveis no mercado, o filtro de Greenfield é o mais popular. Essa popularidade se deve não só à longa e extensa experiência com o seu uso, mas também aos baixos índices de complicações ${ }^{1,2}$. Seu desenho permite que, mesmo

\begin{abstract}
This study presents preliminary results obtained from a new permanent filter, based on Greenfield's filter design, with prolongations on three of six struts to stabilize it centrally in the vena caval lumen. The preliminary clinical evaluation of the filter with regard to feasibility, efficacy and safety is reported. From August 2004 to December 2006, 15 vena cava filters were deployed in nine men and six women, who ranged in age from 38 to 79 years (mean, 57.8 years). The approach used was always transjugular. Indications for filter placement were proximal deep venous thrombosis with a contraindication to anticoagulation in 12 patients; hemorrhagic complications with anticoagulation in two patients; and pulmonary embolism, despite adequate anticoagulation in one patient. New vena cava filters were evaluated for releasing, tilting, malpositioning and caval perforation. Follow-up included assessment of access site thrombosis and filter migration, recurrent venous thromboembolism, and caval thrombosis by duplex ultrasound. No patient received anticoagulants in the follow-up. In all patients the filter was successfully released, with no malpositioning, tilting, perforation or access thrombosis. The patients were followed for 3 to 23 months ( mean $=11$ months). No patient developed recurrent venous thromboembolism. No other patients developed inferior vena cava thrombosis or filter migration. Death occurred in seven patients, all related to baseline illness. This preliminary study suggests good feasibility and safety of the new filter up to the observation period.
\end{abstract}

Keywords: Vena cava filters, prosthesis design, pulmonary embolism/prevention, control, venous thrombosis.

após $70 \%$ do seu cone ter sido preenchido por um trombo, ainda existam $50 \%$ de área para a passagem do fluxo sangüíneo ${ }^{3}$. Várias séries clínicas mostraram mortalidade operatória em torno de $0,4 \%$ associada a seu uso $^{2}$. Revisões recentes como a de Kinney ${ }^{4}$, comparando os filtros de Greenfield ${ }^{\circledR}$, Ninho de Pássaro ${ }^{\circledR}$,

1. Livre-docente. Professor adjunto, Departamento de Cirurgia e Ortopedia, Faculdade de Medicina, Universidade Estadual Paulista (UNESP), Botucatu, SP.

2. Doutor. Professor assistente. Departamento de Cirurgia e Ortopedia, Faculdade de Medicina, UNESP, Botucatu, SP.

Este estudo recebeu auxílio da Fundação de Amparo à Pesquisa do Estado de São Paulo (FAPESP), processo № 03/10854-5.

Não foram declarados conflitos de interesse associados à publicação deste artigo.

Artigo submetido em 26.12.07, aceito em 18.05.08.

J Vasc Bras. 2008;7(3): 282-288.

Copyright@ 2008 by Sociedade Brasileira de Angiologia e de Cirurgia Vascular 
Simon Nitinol ${ }^{\circledR}$ e Vena-Tech ${ }^{\circledR}$, mostraram que o filtro de Greenfield foi o que apresentou menores índices de trombose da veia cava $(3,6,3,9,7,7$ e $11,2 \%$, respectivamente). Outras revisões ${ }^{5}$ mostraram freqüências baixas e similares de migração na veia cava $(5,3,1,9,2,2$ e 8,3\%, respectivamente) e de embolia pulmonar recorrente ( $0 \mathrm{a}$ 9,0 a $4,2,0$ a 5,3 e 0 a $8 \%$, respectivamente).

Embora esteja associado a freqüências menores de trombose de veia cava, o filtro de Greenfield pode provocar outras complicações. Foram relatados, além dos aspectos comentados, trombose da veia de acesso ao implante em cerca de $20 \%$ dos $\operatorname{casos}^{6}$, implantação errônea em veia ilíaca, renal ou aurícula direita ${ }^{7,8}$, migração para a aurícula direita ${ }^{9}$, fratura das hastes ${ }^{10}$, extrusão das hastes ${ }^{11}$ com perfuração de vísceras ${ }^{12}$ e inclinação do filtro em relação ao eixo da veia cava ${ }^{13}$.

A inclinação do filtro de Greenfield na veia cava não é uma complicação freqüente, ocorrendo entre 5,5 a 10\% dos $\operatorname{casos}^{14,15}$. Entretanto, quando ocorre, está associada à perda da eficiência na captura de êmbolos ${ }^{3,16}$, além de favorecer a extrusão das hastes ${ }^{17}$, as quais, por sua vez, podem provocar a perfuração de estruturas vizinhas à veia cava, como ureter, vísceras e aorta ${ }^{18}$.

Visando diminuir esse tipo de complicação, propusemos uma modificação no desenho do filtro de Greenfield, prolongando três das suas seis hastes, de modo que fosse liberado sempre alinhado ao eixo da veia cava. O protótipo foi avaliado experimentalmente, com sucesso ${ }^{7}$.

O objetivo do presente estudo foi implantar um novo filtro de veia cava inferior em pacientes com indicação de implante e avaliar sua liberação e as seguintes freqüências: inclinação do filtro, trombose de veia cava, migração e tromboembolismo venoso (TEV) recorrente. Neste artigo, são apresentados os resultados preliminares.

\section{Métodos}

Foi feito um estudo observacional de série de casos incluindo 15 pacientes consecutivos com TEV e indicação de colocação de filtro de veia cava entre agosto de 2004 e dezembro de 2006. O estudo foi aprovado pelo Comitê de Ética em Pesquisa da instituição e pela Comissão Nacional de Ética em Pesquisa (CONEP). Os pacientes foram informados sobre os objetivos do estudo e os riscos envolvidos; o termo de consentimento livre e esclarecido foi lido e assinado por todos os pacientes.

Os critérios de inclusão para implante do filtro foram:

- Ter idade superior a 18 anos.

- Ser paciente portador de TEV com contra-indicação ao uso de anticoagulantes, como por exemplo: doenças hemorrágicas, cirurgias ou traumas cranioencefálicos, metástases cerebrais, acidentes vasculares cerebrais recentes (até 4 a 6 semanas), hematúria, operações de grande porte recentes (até 7 a 10 dias) com descolamentos extensos, úlcera péptica ativa com sangramento, varizes de esôfago, plaquetopenia importante $(<50.000)$, etc.

- Ser paciente com dificuldade de controle do anticoagulante ou que apresenta complicações hemorrágicas decorrentes de seu uso.

- Casos em que a anticoagulação bem controlada falhou em prevenir a ocorrência de embolia pulmonar.

- Ser paciente submetido à embolectomia pulmonar por embolia maciça.

Os critérios de exclusão foram:

Não concordância do paciente em participar do estudo. Mulheres grávidas (devido à exposição a raio X).

Trombose de veia cava inferior, com indicação de colocação de filtro em veia cava supra-renal.

Necessidade de implante do filtro por via femoral.

Diâmetro da veia cava $>28 \mathrm{~mm}$ e anomalias anatômicas.

Tal qual no trabalho experimental prévio, os filtros modificados foram fabricados na oficina da instituição, utilizando-se fios redondos de aço inoxidável duro e elástico, 316-L com 0,45 mm de diâmetro, para as hastes, reunidas firmemente em ápices também do mesmo aço inoxidável (Figura 1). Os aplicadores foram produzidos na oficina com $12 \mathrm{~F}$ de diâmetro externo e bainhas introdutoras de $90 \mathrm{~cm}$ de comprimento. Cada aplicador foi testado ex vivo, colocando o filtro e liberando-o por 10 vezes. Os filtros e aplicadores foram esterilizados pela técnica Sterrad ${ }^{\circledR}$, no Centro de Esterilização de Materiais do Hospital das Clínicas de Botucatu. 


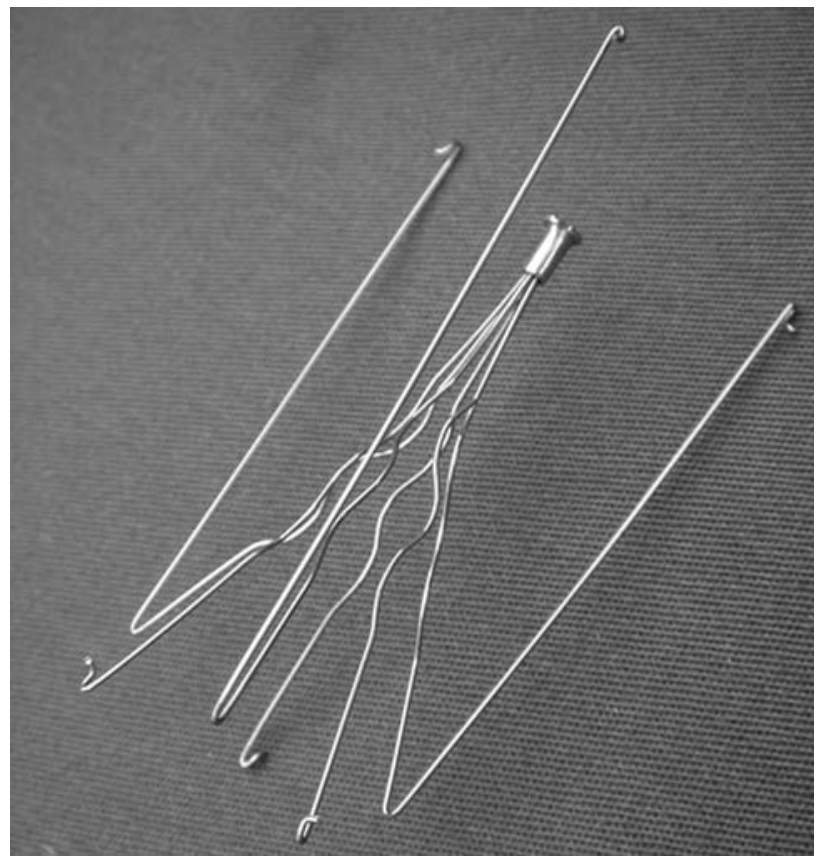

Figura 1 - Novo modelo de filtro permanente de veia cava

\section{Técnica de implante}

A técnica de implante foi feita por via jugular e obedeceu à mesma seqüência padronizada para o filtro de Greenfield $^{19}$.

\section{Avaliações operatórias e recomendações}

Através da cavografia, observou-se o posicionamento do filtro, a perviedade da veia cava, a inclinação do filtro e as perfurações da veia cava.

As avaliações seguintes previstas para este estudo seguiram a rotina do serviço e foram as mesmas necessárias para pacientes que recebem filtros comerciais. Durante a primeira semana de pós-operatório, o local da punção foi inspecionado diariamente, observando-se hematomas, sinais inflamatórios, edemas localizados e infecções. Caso a veia femoral tivesse sido utilizada como via de acesso para se fazer a cavografia, a região inguinal seria também inspecionada diariamente.

$\mathrm{Na}$ alta, os pacientes foram orientados para o uso diário de meia elástica e agendamento semestral do exame de ultra-som da veia cava e membros inferiores. Nenhum paciente recebeu anticoagulantes.

\section{Seguimento a longo prazo}

Os pacientes foram alertados e orientados a se comunicar com a equipe do hospital caso apresentassem, fora da agenda de consultas, manifestações de recorrência de tromboembolismo pulmonar (TEP). Em qualquer momento do estudo que o paciente porventura viesse a manifestar sinais clínicos de trombose venosa ou embolia pulmonar recorrentes, esse diagnóstico seria confirmado pelo exame de ultra-som ou cintilografia pulmonar, respectivamente. A cada 6 meses, a perviedade da veia cava e a migração do filtro seriam avaliados por ultra-som.

\section{Análise estatística}

Foram feitas somente análises descritivas de freqüências e de tendência central.

\section{Resultados}

As indicações para o implante dos filtros foram: trombose venosa proximal com contra-indicação para anticoagulação em 12 pacientes, complicações hemorrágicas decorrentes de anticoagulação em dois pacientes e embolia pulmonar, apesar de anticoagulação adequada, em um paciente. Todos os filtros foram liberados com sucesso, sem intercorrências, em posição adequada e sem inclinação (Figura 2). Não foram observados casos de hematoma, sinais inflamatórios, edema localizado e infecção no sítio de punção. Durante os 30 dias após o procedimento, quatro pacientes vieram a óbito, sendo todos os casos vinculados a doenças de base graves e de prognóstico reservado (Tabela 1). Autópsia foi realizada em dois pacientes (Tabela 1, casos 2 e 4); em nenhum dos quatro casos, houve relação entre o óbito e o filtro.

O período de seguimento variou entre 3 e 23 meses (média de 11 meses). Quatro pacientes perderam seguimento, não tendo sido possível contato por telefone ou através das prefeituras. Não houve, neste período, sinais e sintomas de recorrência de trombose venosa ou embolia pulmonar entre os demais pacientes. Através de exame ultra-sonográfico, não se observou trombose de veia cava ou migração do filtro em seis pacientes que fizeram os exames seriados (Figura 3). Os casos de óbito com autópsia também não apresentaram essas alterações e recorrência de TEP. Os óbitos deste período (após 
Tabela 1 - Dados demográficos, comorbidades e evolução dos pacientes que receberam implante do novo filtro

\begin{tabular}{|c|c|c|c|c|c|c|}
\hline Caso & Sexo & Idade & Data & Comorbidades & $\begin{array}{c}\text { Último } \\
\text { ultra-som }\end{array}$ & Desfecho \\
\hline 1 & M & 49 & $24 / 08 / 2004$ & Chagas + ICC & - & Óbito (2 dias), ICC \\
\hline 2 & M & 67 & $25 / 05 / 2005$ & $\begin{array}{l}\text { Glioblastoma }+ \\
\text { neurocirurgia }\end{array}$ & 12 meses & $\begin{array}{l}\text { Óbito ( } 13 \text { meses), } \\
\text { tumor cerebral }\end{array}$ \\
\hline 3 & $\mathrm{~F}$ & 65 & $17 / 08 / 2005$ & AVC recente & 18 meses & Sem queixas \\
\hline 4 & $\mathrm{~F}$ & 49 & $25 / 10 / 2005$ & $\begin{array}{l}\text { Transplante renal + } \\
\text { infecção por } \\
\text { citomegalovírus + } \\
\text { disenteria }\end{array}$ & - & $\begin{array}{l}\text { Óbito ( } 28 \text { dias), } \\
\text { colite } \\
\text { citomegalovírus }\end{array}$ \\
\hline 5 & M & 69 & $03 / 11 / 2005$ & $\begin{array}{l}\text { Alcoolismo + } \\
\text { hematoma subdural } \\
+ \text { neurocirurgia }\end{array}$ & 3 meses & Perdeu seguimento \\
\hline 6 & M & 73 & $16 / 01 / 2006$ & Úlcera gástrica & 23 meses & Sem queixas \\
\hline 7 & M & 79 & $17 / 01 / 2006$ & $\begin{array}{l}\text { Câncer de cólon + } \\
\text { colectomia }\end{array}$ & - & $\begin{array}{l}\text { Perdeu seguimento } \\
\text { após } 2 \text { meses }\end{array}$ \\
\hline 8 & M & 66 & $06 / 02 / 2006$ & $\begin{array}{l}\text { AVC hemorrágico + } \\
\text { neurocirurgia }\end{array}$ & - & Perdeu seguimento \\
\hline 9 & $\mathrm{~F}$ & 38 & $20 / 04 / 2006$ & $\begin{array}{l}\text { Tumor cerebral + } \\
\text { neurocirurgia }\end{array}$ & 3 meses & $\begin{array}{l}\text { Óbito ( } 8 \text { meses), } \\
\text { tumor cerebral }\end{array}$ \\
\hline 10 & M & 49 & $22 / 06 / 2006$ & $\begin{array}{l}\text { Dissecção aguda de } \\
\text { aorta }\end{array}$ & - & Perdeu seguimento \\
\hline 11 & $\mathrm{~F}$ & 55 & 07/07/2006 & $\begin{array}{l}\text { Câncer de pâncreas } \\
\text { e de útero } \\
\text { inoperáveis + } \\
\text { plaquetopenia }\end{array}$ & - & $\begin{array}{l}\text { Perdeu seguimento } \\
\text { após } 1 \text { mês de } \\
\text { radioterapia }\end{array}$ \\
\hline 12 & $\mathrm{~F}$ & 49 & 08/06/2006 & $\begin{array}{l}\text { Câncer gástrico }+ \\
\text { hemorragia digestiva }\end{array}$ & - & $\begin{array}{l}\text { Óbito ( } 2 \text { dias), } \\
\text { hemorragia digestiva }\end{array}$ \\
\hline 13 & M & 50 & $08 / 12 / 2006$ & $\begin{array}{l}\text { SAAF catastrófica + } \\
\text { hemorragia com } \\
\text { anticoagulante }\end{array}$ & - & $\begin{array}{l}\text { Óbito ( } 2 \text { meses), } \\
\text { SAAF catastrófica }\end{array}$ \\
\hline 14 & M & 55 & $28 / 12 / 2006$ & AVC + pneumonia & - & $\begin{array}{l}\text { Óbito (1 dia), } \\
\text { taquiarritmia } \\
\text { supraventricular + } \\
\text { convulsões }\end{array}$ \\
\hline 15 & $\mathrm{~F}$ & 55 & $15 / 01 / 2007$ & AVC & 7 meses & Sem queixas \\
\hline
\end{tabular}

ICC = insuficiência cardíaca congestiva; $\mathrm{AVC}=$ acidente vascular cerebral; SAAF = síndrome do anticorpo antifosfolípide.

30 dias de pós-operatório) foram três, também vinculados às moléstias de base (Tabela 1).

\section{Discussão}

O presente estudo mostra a implantação com sucesso de um novo modelo de filtro de veia cava inferior desenvolvido na Universidade Estadual Paulista (UNESP). O modelo foi baseado no filtro de Greenfield, visando corrigir uma das intercorrências deste filtro que é o implante descentralizado, que leva à perda de sua eficácia. Neste estudo piloto, não se observaram intercorrências, tanto com relação à falha na liberação do filtro como no que diz respeito à implantação descentralizada. No seguimento, os filtros permaneceram centralizados e não houve deslocamento, migração, perfuração e trombose da veia cava. Em relação à evolução dos 


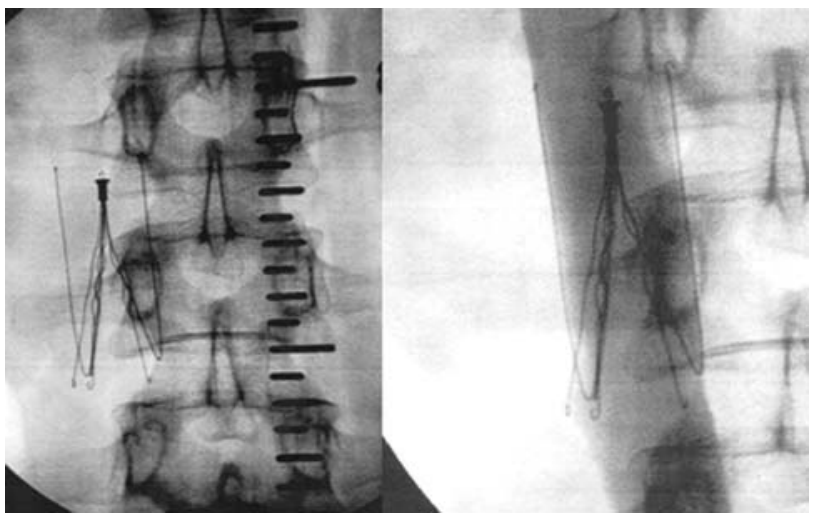

Figura 2 - Cavografia mostrando posicionamento adequado do novo filtro, sem inclinação

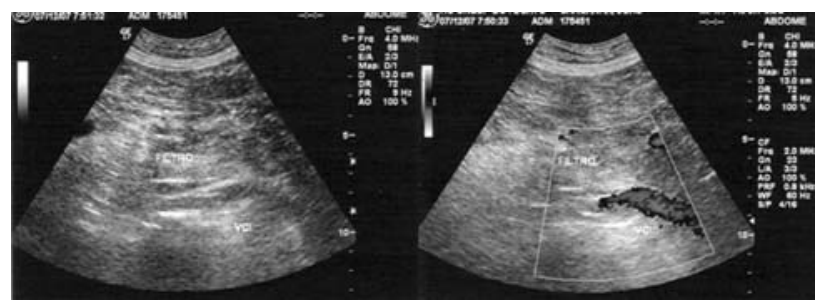

Figura 3 - Imagem ultra-sonográfica da veia cava pérvia com novo filtro após 23 meses

pacientes, houve quatro mortes no primeiro mês, devido a doenças de base, além de perda de seguimento de quatro pacientes. Portanto, pudemos notar que houve em geral boa evolução nos pacientes sem comorbidades importantes.

Os quatro pacientes que faleceram em até 30 dias após o procedimento estavam em situação clínica bastante grave (Tabela 1), não tendo sido o implante do filtro o responsável por esse desfecho; os três pacientes que faleceram ao longo do período de estudo, por sua vez, também tinham comorbidades importantes. Como ressaltado por Hingorani et al., a moléstia de base é muitas vezes mais importante em termos de mortalidade do que a trombose venosa em $\mathrm{si}^{20}$. A indicação de filtros para pacientes muito graves acaba dando a impressão de mortalidade elevada vinculada ao procedimento, o que nem sempre é verdade, uma vez que o prognóstico desses pacientes é reservado. Por exemplo, em pacientes com câncer e metástases, ou clinicamente muito instáveis, pode ser eventualmente questionável a realização desse procedimento, devido ao alto custo vinculado ${ }^{21}$.
A mortalidade operatória ligada diretamente ao procedimento de implante de filtros é em geral baixa, sendo estimada em $0,3 \%$ com o filtro de Greenfield ${ }^{1,22}$. Em longo prazo, perto de $43 \%$ dos pacientes com filtros acabam falecendo por várias causas ${ }^{19}$. Em nossa pequena amostra, $46,6 \%$ dos pacientes vieram a falecer.

O melhor exame para atestar a perviedade da veia cava seria a cavografia. Entretanto, trata-se de exame invasivo, que requer punção da veia femoral, cateterismo iliocaval, injeção de contraste iodado e uso de radiação. O exame ultra-sonográfico, por outro lado, não é invasivo, mas está sujeito a dificuldade, dependendo das condições do exame. Embora pedidos de ultra-som tenham sido feitos a cada 6 meses para todos os pacientes, vários deles não compareceram para realização dos exames e quatro pacientes não mais apareceram no hospital e não puderam ser encontrados apesar de busca ativa (Tabela 1). Todos os seis pacientes que chegaram a fazer pelo menos um exame ultrasonográfico, estavam com as veias cavas pérvias e sem migração do filtro.

A oclusão da veia cava após implante pode ocorrer com qualquer tipo de filtro. Em levantamento feito por Streiff $^{23}$ e Kinney ${ }^{4}$, essas oclusões variaram de acordo com o tipo de filtro implantado, sendo os filtros Ninho de Pássaro e Greenfield os que tiveram menores taxas de oclusão da veia cava em junção de dados de série de casos.

O novo filtro mostrou também facilidade de aplicação, liberação completa em todos os casos, bom posicionamento na veia cava, sem inclinação e sem problemas no sítio de acesso pela veia jugular, apesar da limitação do seu perfil (12 F), similar ao filtro de Greenfield. O prolongamento das três hastes (Figura 1) realmente tende a estabilizar o mesmo no eixo longitudinal da veia cava, sem facilitar a migração ou recorrência de TEV. Devido ao desenho similar ao filtro de Greenfield e aos resultados do estudo experimental, é possível que nosso filtro apresente taxas de oclusão de veia cava similares ao de Greenfield, embora o novo modelo tenha um pouco mais de material. No entanto, é preciso estudar o seu comportamento a longo prazo com um número maior de pacientes para conclusões definitivas. 
A possibilidade de migração é outro aspecto técnico importante com relação aos filtros. No levantamento de Streiff $^{23}$, a migração foi menos evidente com os filtros Ninho de Pássaro e Simon Nitinol, intermediária com o filtro de Greenfield e maior com o filtro Vena-Tech, modelo tradicional.

Finalmente, um importante aspecto a ser discutido é a recorrência de TEP após colocação do filtro. As taxas de recorrência de embolia pulmonar são similares entre os filtros, porém taxas de TVP recorrente variam entre 6 e $30 \%{ }^{24}$. O único trabalho controlado prospectivo abordando esse aspecto foi feito por Decousus et al. ${ }^{19,25}$. Nesse trabalho, 400 pacientes foram randomizados para receber filtro de veia cava + anticoagulantes ou somente anticoagulantes. As taxas de recorrência de embolia pulmonar em 12 dias de seguimento foram menores nos pacientes que receberam o filtro + anticoagulantes em relação aos anticoagulados sem filtro. Porém, a longo prazo ( 2 e 8 anos), a recorrência tanto de TVP como de embolia pulmonar foi estatisticamente maior no grupo que recebeu filtro + anticoagulantes. Neste trabalho, não foram analisados pacientes que receberam filtros e não foram anticoagulados, mas os dados apresentados sugerem que seria importante a manutenção da anticoagulação nos pacientes com filtro, tão logo pudessem receber os anticoagulantes. Os resultados observados por Decousus et al. no seguimento de 8 anos foram similares $^{19}$. Os nossos pacientes não receberam anticoagulantes após o filtro. Entretanto, deve-se ressaltar que, pelo menos quanto ao filtro de Greenfield, as taxas de oclusão e de recorrência de TEP, foram obtidas, em geral, sem a associação de tratamento anticoagulante ${ }^{21}$.

Em conclusão, nosso filtro não apresentou problemas técnicos durante o procedimento de implante, nem tendência à migração, recorrência ou trombose de veia cava nos poucos pacientes que fizeram avaliações seriadas com ultra-som. Entretanto, em função do pequeno tamanho da amostra, não é possível estabelecer conclusões definitivas. Os resultados preliminares apontam no sentido de potencial eficácia e segurança com o uso no novo filtro, o que favorece a continuidade deste estudo.

\section{Referências}

1. Yoshida WB, Lastoria S. Procedimentos de interrupção venosa na trombose venosa profunda e embolia pulmonar. In: Maffei FHA, Lastoria S, Yoshida WB, Rollo HA, editores. Doenças vasculares periféricas. Rio de Janeiro: Medsi; 2002. vol. 2, p. 1473-85.
2. Greenfield LJ, Michna BA. Twelve-year clinical experience with the Greenfield vena caval filter. Surgery. 1988;104:70612 .

3. Katsamouris AA, Waltman AC, Delichatsios MA, Athanasoulis CA. Inferior vena cava filters: in vitro comparison of clot trapping and flow dynamics. Radiology. 1988;166:361-6.

4. Kinney TB. Update on inferior vena cava filters. J Vasc Interv Radiol. 2003;14:425-40.

5. Streiff MB. Vena caval filters: a comprehensive review. Blood. 2000;95:3669-77.

6. Aswad MA, Sandager GP, Pais SO, et al. Early duplex scan evaluation of four vena caval interruption devices. J Vasc Surg. 1996;24:809-18.

7. Messmer JM, Greenfield LJ. Greenfield caval filters: longterm radiographic follow-up study. Radiology. 1985;156:613-8.

8. Abad C, Pomar JL, Morales MA. [Accidental opening of a Greenfield filter at the right atrium]. Angiologia. 1990;42:182-4.

9. Greenfield LJ, Peyton R, Crute S, Barnes R. Greenfield vena caval filter experience: late results in 156 patients. Arch Surg. 1981;116:1451-6.

10. Lang W, Schweiger H, Fietkau R, Hofmann-Preiss K. Spontaneous disruption of two Greenfield vena caval filters. Radiology. 1990;174:445-6.

11. Donaldson MC, Wirthlin LS, Donaldson GA. Thirty-year experience with surgical interruption of the inferior vena cava for prevention of pulmonary embolism. Ann Surg. 1980;191:367-72.

12. Kupferschmid JP, Dickson CS, Townsend RN, Diamond DL. Small-bowel obstruction from an extruded Greenfield filter strut: an unusual late complication. J Vasc Surg. 1992;16:113-5.

13. Rogers FB, Shackford SR, Ricci MA, Huber BM, Atkins T. Prophylactic vena cava filter insertion in selected high-risk orthopaedic trauma patients. J Orthop Trauma. 1997;11:26772 .

14. Greenfield LJ, Proctor MC, Cho KJ, et al. Extended evaluation of the titanium Greenfield vena caval filter. J Vasc Surg. 1994;20:458-64; discussion 464-5.

15. Rogers FB, Strindberg G, Shackford SR, et al. Five-year follow-up of prophylactic vena cava filters in high-risk trauma patients. Arch Surg. 1998;133:406-11; discussion 412.

16. Kinney TB, Rose SC, Weingarten KE, Valji K, Oglevie SB, Roberts AC. IVC filter tilt and asymmetry: comparison of the over-the-wire stainless-steel and titanium Greenfield IVC filters. J Vasc Interv Radiol. 1997;8:1029-37.

17. Yoshida WB, Sequera J, De Abreu Maffei FH. Long-term histopathologic evaluation of inferior vena cava after modified Greenfield filter implantation. Experimental study in sheep. Int Angiol. 2004;23:170-6.

18. Dorfman GS. Percutaneous inferior vena caval filters. Radiology. 1990;174(3 Pt 2):987-92.

19. PREPIC Study Group. Eight-year follow-up of patients with permanent vena cava filters in the prevention of pulmonary embolism: the PREPIC (Prevention du Risque d'Embolie Pulmonaire par Interruption Cave) randomized study. Circulation. 2005; 112:416-22. 
20. Hingorani A, Ascher E, Markevich N, et al. Risk factors for mortality in patients with upper extremity and internal jugular deep venous thrombosis. J Vasc Surg. 2005;41:476-8.

21. Rectenwald JE, Greenfield LJ, Henke PK, Proctor MC, Wakefileld TW. Vena caval interruption procedures. In: Rutherford RB, editor. Vascular surgery. Philadelphia: Elsevier-Saunders; 2005. vol. 2, p. 2198-216.

22. Yoshida WB, Rollo HA, Lastoria S, Maffei FHA. Interrupção venosa como tratamento de exceção na trombose venosa profunda e embolia pulmonar. Cir Vasc Angiol. 1987;3:23-9.

23. Streiff MB. Vena caval filters: a review for intensive care specialists. J Intensive Care Med. 2003;18:59-79.

24. British Committee for Standards in Haematology Writing Group, Baglin TP, Brush J, Streiff M. Guidelines on use of vena cava filters. Br J Haematol. 2006;134:590-5.
25. Decousus H, Leizorovicz A, Parent F, et al. A clinical trial of vena caval filters in the prevention of pulmonary embolism in patients with proximal deep-vein thrombosis. Prevention du Risque d'Embolie Pulmonaire par Interruption Cave Study Group. N Engl J Med. 1998;338:409-15.

Correspondência:

Winston Bonetti Yoshida

Departamento de Cirurgia e Ortopedia

Faculdade de Medicina de Botucatu - UNESP

CEP 18618-970 - Botucatu, SP

E-mail: winston@fmb.unesp.br 